\title{
Evaluation des dégâts des insectes ravageurs du chou pomme (Brassica oleracea var. capitata L.) à Korhogo, nord de la Côte d'Ivoire
}

\author{
San-Whouly Mauricette OUALI N'GORAN ${ }^{1}$, Madeleine Adjoua KOUASSI ${ }^{1,2 *}$ et \\ Adama COULIBALY ${ }^{1,3}$ \\ ${ }^{1}$ UFR Biosciences, Université Félix HOUPHOUËT-BOIGNY, p 582 Abidjan 22, Abidjan, Côte d'Ivoire. \\ ${ }^{2}$ Institut de Gestion Agropastorale (IGA), Université Peleforo GON COULIBALY, BP 1328 Korhogo, \\ Côte d'Ivoire. \\ ${ }^{3}$ UFR Sciences Biologiques, Université Peleforo GON COULIBALY, Korhogo, BP 1328 Korhogo, \\ Côte d'Ivoire. \\ *Auteur correspondant; E-mail : madeleinkouassi@gmail.com; Tél. + 22507718492
}

\begin{tabular}{lll}
\hline Received: 07-09-2020 & Accepted: 18-02-2021 & Published: 28-02-2021 \\
\hline
\end{tabular}

\section{RESUMÉ}

Le chou fait partie des principales cultures maraîchères pratiquées en Côte d'Ivoire. Sa production est cependant limitée par des problèmes parasitaires entomologiques mal maîtrisés. Cette étude a été réalisée sur trois périmètres maraîchers du département de Korhogo, de juillet 2016 à juin 2017. Elle vise à évaluer les niveaux d'attaques des insectes ravageurs et à déterminer leur impact sur la culture. De façon spécifique, il s'est agi d'identifier les espèces d'insectes collectés sur les plants, de décrire leurs dégâts et de déterminer l'intensité de leurs attaques à travers un indice (I) compris entre $0 \%$ et $100 \%$. Les observations ont révélé la présence de quinze espèces d'insectes. Les larves et/ou les adultes de ces insectes attaquaient les plants de chou. Les dégâts affectent les feuilles, le bourgeon apical, la pomme et la tige. Les espèces Hellula undalis et Plutella xylostella ont été identifiées comme les ravageurs majeurs du chou car elles ont attaqué respectivement 57,04\% des plants avec une forte intensité $(\mathrm{I}=38,89 \pm 21,45 \%)$ et $88,15 \%$ des plants avec une très forte intensité $(\mathrm{I}=56,11 \pm$ $18,87 \%$ ). La connaissance de ces ravageurs majeurs et de leurs dégâts pourrait contribuer à une meilleure gestion de ces nuisibles.

(C) 2020 International Formulae Group. All rights reserved.

Mots clés : Chou, intensité des attaques, Hellula undalis, Plutella xylostella, Côte d'Ivoire.

\section{Assessment of damage caused by insect pests on cabbage (Brassica oleracea var. capitata L.) in Korhogo, northern Côte d'Ivoire}

\begin{abstract}
Cabbage is one of the main vegetable crops grown in Côte d'Ivoire. However, its production is limited by the poor insect management. This study was carried out through three vegetable areas in the division of Korhogo from July 2016 to June 2017. It aims to assess the damage caused by insect and determine their impact on the crop. Specifically, it involved identifying the insect species collected from the plants, describing their damage and determining the intensity of their attacks using an index between $0 \%$ and $100 \%$. Observations
\end{abstract}


revealed fifteen insect species. The larvae and/or adults of these insects attacked cabbage plants. The damage affects the leaves, the apical bud, the apple and the stem. The species Hellula undalis and Plutella xylostella were identified as the major pests of cabbage because they attacked respectively $57.04 \%$ of the plants with high intensity $(\mathrm{I}=38.89 \pm 21.45 \%)$ and $88.15 \%$ of the plants with a very high intensity $(\mathrm{I}=56.11 \pm 18.87 \%)$. Knowledge of these major pests and their domages could contribute to better management of these pests.

(C) 2020 International Formulae Group. All rights reserved.

Keywords: Cabbage, intensity of attacks, Hellula undalis, Plutella xylostella, Côte d'Ivoire.

\section{INTRODUCTION}

Les cultures maraîchères représentent, en Côte d'Ivoire, une activité économique capitale pour les populations qui l'exercent. Ce sont plus de $83 \%$ des producteurs qui supportent les besoins de leurs familles grâce à cette activité (Soro et al., 2018). Bien que les cultures maraîchères soient importantes du point de vue économique et alimentaire, leur mode de culture, en revanche, est loin de répondre aux normes écologiques de production (Tano et al., 2012 ; Wognin et al., 2013; Akessé et al., 2015). En effet, des résidus de pesticides ont été retrouvés dans les récoltes ainsi que dans les sols et l'eau des parcelles maraîchères à des doses supérieures à la limite maximale de résidus (Akessé, 2014 ; Mambe-Ani et al., 2019 ; Gueye et al., 2020). Cette accumulation de résidus résulte de l'utilisation abusive des pesticides chimiques de synthèse. Ces derniers sont utilisés pour faire face à la forte pression parasitaire infligée surtout par les insectes ravageurs (Assi et al., 2018 ; Koné et al., 2019 ; Tuo et al., 2019). Sur le chou qui est l'une des cultures maraîchères les plus importantes en Côte d'Ivoire, 25 espèces d'insectes ravageurs ont été recensées avec une prédominance des Lépidoptères Plutella xylostella, Hellula undalis et Spodoptera littoralis (Douan et al., 2013 ; Tuo et al., 2017 ; Kouassi et al., 2019). Pour limiter les traitements insecticides sur ces cultures et définir des seuils de nuisibilité, Douan et al. (2013) ont réalisé une étude de dépistage basée sur le piégeage des adultes et le comptage direct des larves de ces trois Lépidoptères. Ce dépistage n'est pas toujours aisé, surtout pour des larves qui ont des mœurs nocturnes ou qui vivent cachées à l'intérieur de certains organes végétatifs des plants. Une approche symptomatique s'avère nécessaire dans ces cas pour détecter la présence des insectes ravageurs. Cette approche, basée sur la reconnaissance des dégâts, suggère une caractérisation préalable des attaques afin de déterminer les ravageurs dont les dégâts méritent l'adoption d'une méthode de lutte appropriée et ciblée. L'objectif de cette étude est d'identifier les principaux insectes ravageurs du chou et de déterminer l'intensité de leurs dégâts. L'évaluation des dégâts permettra d'éviter des traitements excessifs, néfastes pour l'environnement et la santé humaine.

\section{MATÉRIEL ET MÉTHODES Site d'étude}

L'étude s'est déroulée dans le département de Korhogo, au nord de la Côte d'Ivoire. Le climat est de type tropical soudanais avec une grande saison pluvieuse et une grande saison sèche. Les températures moyennes varient entre 24 et $33{ }^{\circ} \mathrm{C}$ avec une moyenne pluviométrique annuelle qui oscille entre $1100 \mathrm{~mm}$ et $1600 \mathrm{~mm}$. Le sol a une texture sablo-limoneuse (BNETD, 2007). Trois sites ont été choisis dans la zone urbaine et péri-urbaine de la ville de Korhogo. Le premier site est situé dans le village de Waraniéné, à $l^{\prime}$ est de la ville $\left(9^{\circ} 26^{\prime} \mathrm{N}, 5^{\circ} 40^{\prime} \mathrm{W}\right)$. Le second se trouve au nord-est de la ville, sur les berges du barrage du quartier Mongaha $\left(9^{\circ} 28^{\prime} \mathrm{N}\right.$, $\left.5^{\circ} 39^{\prime} \mathrm{W}\right)$. Ce sont, tous deux, des périmètres maraîchers. Ils ont été choisis parce qu'ils renferment les surfaces cultivées en chou les plus importantes (Kouassi et al., 2019). La culture du chou y est pratiquée toute l'année. Quant au troisième site, il est localisé au carré potager de l'Université Peleforo GON COULIBALY, au sud-est de la ville $\left(9^{\circ} 26^{\prime} \mathrm{N}\right.$, 
$\left.5^{\circ} 38^{\prime} \mathrm{W}\right)$. C'est un site d'expérimentation agricole.

\section{Collecte des données \\ Dispositif expérimental}

Les expérimentations ont été conduites sur trois cycles culturaux successifs (juillet octobre 2016 ; novembre 2016 - février 2017 ; mars - juin 2017) de façon à couvrir les différentes conditions climatiques. Une parcelle expérimentale de $55 \mathrm{~m}^{2}(10 \mathrm{~m} \times 5,5 \mathrm{~m})$ a été mise en place sur chacun des sites. Elle a comporté 3 parcelles élémentaires de $11 \mathrm{~m}^{2}$ chacune $(5,5 \mathrm{~m} \times 2 \mathrm{~m})$. Deux parcelles élémentaires consécutives étaient séparées de 2 $\mathrm{m}$. Au niveau de chaque parcelle élémentaire, les plants ont été disposés sur 3 lignes de $4,5 \mathrm{~m}$ de long séparées les unes des autres par $0,5 \mathrm{~m}$ de distance. Les plants ont également été séparés de $0,5 \mathrm{~m}$. Chaque parcelle élémentaire a été composée de 30 plants parmi lesquels 10 ont été choisis selon une ligne en zigzag pour la collecte des données (Kouassi et al., 2019).

\section{Collecte et identification des insectes ravageurs}

Afin d'identifier les insectes ravageurs des plants de chou, des observations sur les plants ont été effectuées une fois en pépinière (21 jours après le semis) et cinq fois après le repiquage $(14,35,56,77$ et 98 jours après repiquage). Tous les organes aériens ont été inspectés. Un plant a été dit attaqué lorsqu'il présentait une altération de ses organes (perforations, pourriture partielle ou totale, déformation). Les insectes causant les altérations ont été capturés et les détériorations ont été décrites. Les ravageurs capturés au stade larvaire ont été mis en élevage jusqu'à l'émergence des adultes. Ils ont ensuite été identifiés à partir de la description de leurs caractères morphologiques à l'aide d'une loupe à main de marque Optico $(6,5 \mathrm{D} ; 2,63 \mathrm{x})$ ou d'un microscope monoculaire de marque MOTIC sous un grossissement de $40 \mathrm{x}$. L'identification des Hémiptères a été faite grâce à la clé de Leclant (1999). Les espèces des autres Ordres ont été identifiées à l'aide de catalogues (Hammes et Putoa, 1986 ; Zahradnik et Chvala, 1989 ; Gourmel,
2014 ; Bellmann, 2015 ; Ramel, 2016 ; Gourmel, 2019).

Détermination de l'intensité des dégâts par ravageur

Détermination du niveau d'attaque

Le niveau d'attaque a été lié au type et au pourcentage d'organes attaqués. Quatre niveaux d'attaque ont été définis en se basant, en partie, sur les estimations de Sall-Sy (2013). Ce sont :

- Faible : moins de 10\% des feuilles sont endommagées ;

- Modéré : 10 à 30\% des feuilles sont endommagées ;

- Grave : plus de 30\% des feuilles sont endommagées ;

- Très grave : la pomme et/ou la tige sont endommagées.

Calcul de l'indice d'intensification des dégâts

Un indice d'intensification des dégâts a été calculé afin de déterminer les espèces dont les dégâts sont préjudiciables à la culture. $\mathrm{Ce}$ calcul a été inspiré de la méthode de Aléne et al. (2006). En fonction des niveaux d'attaques, des pourcentages ont été attribués. Ils ont été estimés à $25 \%$ pour les plants présentant des attaques de niveau faible, $50 \%$ pour ceux de niveau modéré, $75 \%$ et $100 \%$ respectivement pour les attaques de niveaux grave et très grave. L'indice d'intensification des dégâts (I) a été évalué selon la formule ci - dessous :

$\mathrm{I}=[(\mathrm{P} 1 \times 25 \%)+(\mathrm{P} 2 \times 50 \%)+(\mathrm{P} 3 \times 75 \%)+$ $(\mathrm{P} 4 \times 100 \%)] /[\mathrm{P} 0+\mathrm{P} 1+\mathrm{P} 2+\mathrm{P} 3+\mathrm{P} 4]$

$\mathrm{I}$ : indice d'intensification des dégâts par parcelle ;

P0 : nombre de plants sains ;

P1 : nombre de plants présentant des attaques de niveau faible ;

P2 : nombre de plants présentant des attaques de niveau modéré ;

P3 : nombre de plants présentant des attaques de niveau grave ;

P4 : nombre de plants présentant des attaques de niveau très grave.

Les valeurs de l'indice d'intensification des dégâts ont été regroupées en quatre classes établies par Aléne et al. (2006) :

Classe $1: 0 \%<\mathrm{I} \leq 10 \%$, les dégâts sont de faible intensité ; 
Classe $2: 10 \%<\mathrm{I} \leq 30 \%$, les dégâts sont d'intensité moyenne ;

Classe $3: 30 \%<\mathrm{I} \leq 50 \%$, l'intensité des dégâts est forte ;

Classe $4: 50 \%<\mathrm{I} \leq 100 \%$, l'intensité des dégâts est très forte.

Sur la base de cette classification des dégâts, la catégorisation suivante des insectes ravageurs a été proposée :

Les ravageurs mineurs sont ceux dont les dégâts sont de faible intensité ;

Les ravageurs importants sont ceux dont les dégâts sont d'intensité moyenne ;

Les ravageurs majeurs sont ceux dont les dégâts sont d'intensité forte ou très forte.

\section{Analyses statistiques}

Des moyennes et des écarts-types ont été calculés pour le nombre de plants attaqués par niveau d'attaque et pour l'indice d'intensification des dégâts. Ces moyennes ont été soumises à une analyse de variances et comparées avec le test post hoc de Tukey grâce au logiciel SPSS 20.0. Le seuil de probabilité retenu est $\mathrm{p}<0,05$.

\section{RÉSULTATS}

\section{Insectes ravageurs du chou pomme et leurs dégâts}

Quinze espèces ont été recensées sur les plants attaqués. Il s'agit de Plutella xylostella (Linnaeus) (Lepidoptera ; Plutellidae), Hellula undalis (Fabricius) (Lepidoptera ; Crambidae), Hellula phidilealis (Walker) (Lepidoptera; Crambidae), Crocidolomia binotalis (Zeller) (Lepidoptera ; Crambidae), Spodoptera littoralis (Boiduval) (Lepidoptera ; Noctuidae), Trichoplusia ni (Hübner) (Lepidoptera ; Noctuidae), Zonocerus variegatus (Linnaeus) (Orthoptera; Pyrgomorphidae), Pyrgomorpha conica (Olivier) (Orthoptera; Pyrgomorphidae), Henosepilachna elaterii (Rossi) (Coleoptera ; Coccinellidae), Lipaphis erysimi (Kaltenbach) (Homoptera; Aphididae), Myzus persicae (Sulzer) (Homoptera; Aphididae), Lucilia $s p$ (Diptera; Calliphoridae), Musca domestica (Linnaeus) (Diptera ; Muscidae), Sarcophaga $s p$ (Diptera; Sarcophagidae) et Hermetia illucens (Linnaeus) (Diptera ; Stratiomyidae).
Les dégâts des larves de Lépidoptères $P$. xylostella, S. littoralis, C. binotalis, $T$. $n i$ et $H$. phidilealis ont été caractérisés par la perforation des feuilles de chou et de la pomme (Figures 1 et 2). Les attaques ont débuté en pépinière pour $P$. xylostella et $T$. ni. Au niveau de la récolte, les altérations ont été matérialisées par la présence des larves de $C$. binotalis et de $S$. littoralis dans les pommes. Des nymphes de $S$. littoralis ont également été retrouvées dans des galeries creusées dans les pommes. Quant aux Lépidoptères Crambidae $H$. undalis et $H$. phidilealis, leurs attaques ont été traduites par la présence de galeries dans la tige et les nervures principales des feuilles, la destruction du bourgeon apical et l'altération des jeunes pommes de l'intérieur vers l'extérieur (Figure 3).

Les attaques des Orthoptères $Z$. variegatus et $P$. conica ont été caractérisées par la consommation des feuilles à partir des bordures (Figure 4). L'alimentation du Coléoptère $H$. elaterii, quant à elle, a consisté à ronger de façon superficielle la face inférieure des feuilles. En ce qui concerne les pucerons, ces insectes font des piqûres sur les feuilles et les pommes. Ce qui a pour conséquence le retard de croissance des plants.

Les attaques des Diptères Lucilia sp, $M$. domestica, Sarcophaga sp et H. illucens ont été matérialisées par la présence de leurs asticots et de leurs nymphes dans les tissus végétaux en décomposition de la pomme (Figure 5).

\section{Catégorisation des ravageurs selon l'intensité de leurs dégâts}

Les ravageurs du chou ont été responsables d'attaques dont les niveaux ont varié d'une espèce à l'autre. Le nombre moyen de plants attaqués a été compris entre 0 et 3,74 $\pm 2,26$ en fonction du niveau d'attaque (Tableau 1). Les valeurs de l'indice d'intensification des dégâts ont varié de 2,04 \pm $3,73 \%$ à 56,11 $\pm 18,87 \%$ (Tableau 2). Elles ont permis d'identifier huit ravageurs mineurs, cinq ravageurs importants et deux ravageurs majeurs.

\section{Ravageurs mineurs}

Les taux d'attaque des plants des espèces H. elaterii, P. conica, Z. variegatus, $T$. 
ni, Sarcophaga sp, Lucilia sp, H. illucens et $M$. domestica ont été compris entre $6,3 \%$ et $15,55 \%$ (Tableau 2). Le niveau d'attaque a été faible pour $H$. elaterii, $P$. conica, $Z$. variegatus et $T$. $n i$ mais est élevé pour $T$. $n i$ lorsque l'attaque survient en pépinière (Tableau 1). Quant à Sarcophaga sp, Lucilia sp, H. illucens et $M$. domestica, elles ont occasionné des attaques graves mais ces attaques ont concerné moins de $10 \%$ des plants. L'indice d'intensification des dégâts a été faible pour ces huit espèces $(\mathrm{I}<10 \%)$. Les espèces $H$. elaterii $(\mathrm{I}=2,04 \pm 3,73 \%), P$. conica $(\mathrm{I}=3,70$ $\pm 4,92 \%), Z$. variegatus $(\mathrm{I}=3,89 \pm 4,66 \%), T$. $n i(\mathrm{I}=4,07 \pm 5,00 \%)$, Sarcophaga sp $(\mathrm{I}=6,30$ $\pm 7,41 \%)$, Lucilia $s p(\mathrm{I}=7,40 \pm 10,72 \%), \mathrm{H}$. illucens $(\mathrm{I}=7,78 \pm 9,02 \%)$ et $M$. domestica (I $=9,26 \pm 12,65 \%)$ peuvent donc être considérées comme des ravageurs mineurs.

\section{Ravageurs importants}

Les pucerons $M$. persicae et L. erysimi ont attaqué respectivement $23,7 \%$ et $28,15 \%$ des plants en pépinière et en début de culture avec un niveau d'attaque grave. L'indice d'intensification de ces dégâts a été de 17,78 \pm $5,93 \%$ pour $M$. persicae et $21,11 \pm 8,91 \%$ pour L. erysimi (Tableau 2). Ces indices permettent donc de classer ces deux espèces comme des ravageurs importants.

Les larves des Lépidoptères $H$. phidilealis, $C$. binotalis et $S$. littoralis ont causé des dégâts sur plus de $30 \%$ des plants $(36,66 \%$, $32,96 \%$ et $39,63 \%$ respectivement). L'indice

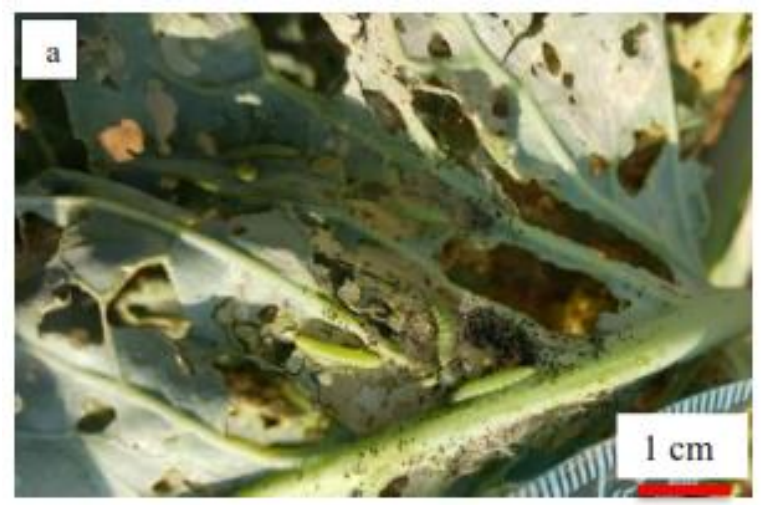

d'intensification des dégâts a été de 21,11 \pm $15,33 \%$ pour $H$. phidilealis, $21,11 \pm 16,60 \%$ pour $C$. binotalis et $21,85 \pm 19,47 \%$ pour $S$. littoralis. Ces trois espèces de Lépidoptères peuvent donc également être considérées comme des ravageurs importants à prendre en compte dans l'élaboration des stratégies de lutte.

\section{Ravageurs majeurs}

L'espèce $P$. xylostella a enregistré le nombre de plants attaqués le plus élevé, quel que soit le niveau d'attaque, avec environ deux plants par parcelle (Tableau 1). Ses dégâts se sont étendus à $88,15 \%$ des plants cultivés avec un indice d'intensification de $56,11 \pm 18,87 \%$. La valeur de l'indice d'intensification des dégâts étant supérieure à $50 \%$, l'intensité des dégâts a été jugée très forte. L'espèce $P$. xylostella responsable de ces dégâts, peut donc être considérée comme un ravageur majeur de la culture du chou pomme à Korhogo.

Les larves de $H$. undalis ont également causé des dégâts de tous les niveaux sur plus de la moitié des plants cultivés $(57,04 \%)$. Les dégâts les plus importants qu'elles ont occasionnés se sont étendus à 23,3\% des plants avec un niveau d'attaque grave, et à $12,9 \%$ des plants avec un niveau d'attaque très grave. La valeur de l'indice d'intensification des dégâts causés par cette espèce a été de 38,89 \pm $21,45 \%$. L'intensité de ces attaques est donc jugée forte ; ce qui fait de $H$. undalis, le second ravageur majeur de la culture du chou.

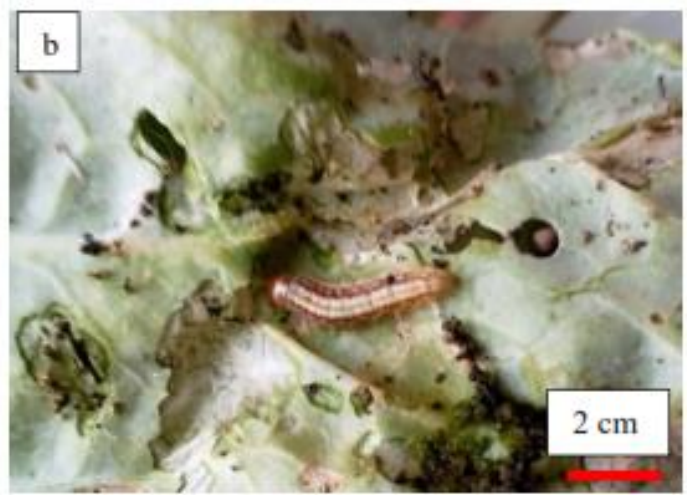

Figure 1 : Feuilles de chou perforées par les larves de Lépidoptères.

a. Larves de Plutella xylostella rongeant la face inférieure d'une feuille de chou ;

b. Larve de Hellula phidilealis rongeant la face inférieure d'une feuille de chou. 

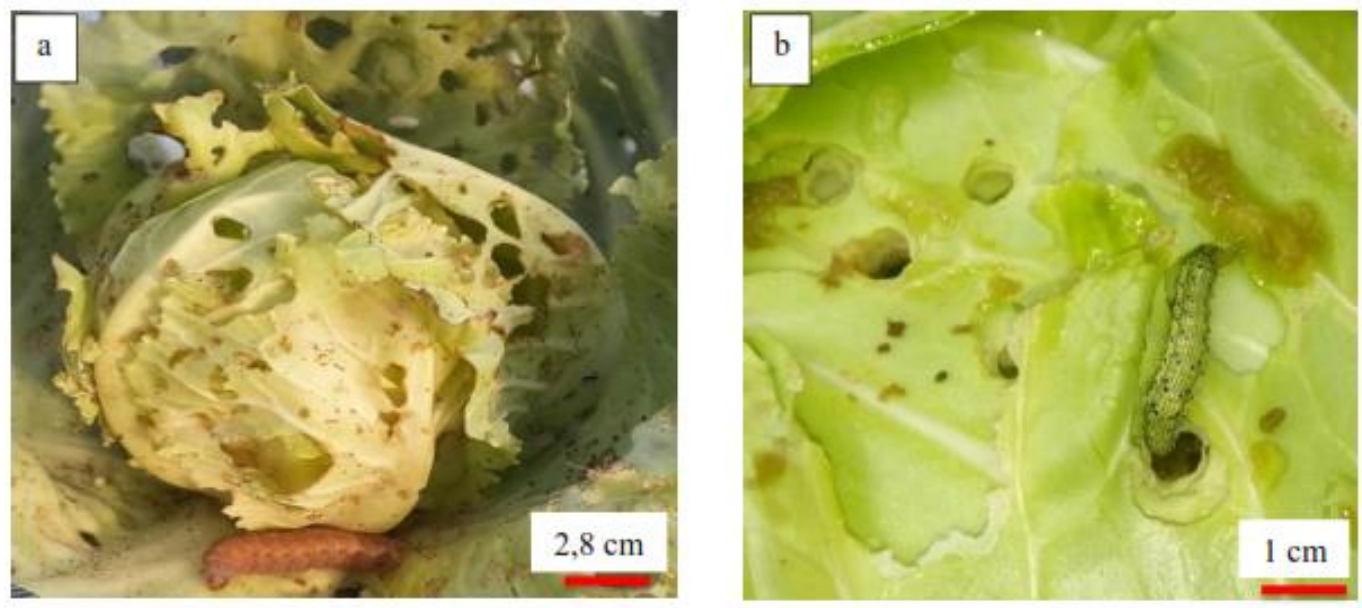

Figure 2 : Pommes de chou perforées par les larves de Lépidoptères.

a. Feuilles superficielles perforées par une larve de Spodoptera littoralis ;

b. Forage d'une pomme par une larve de Crocidolomia binotalis.
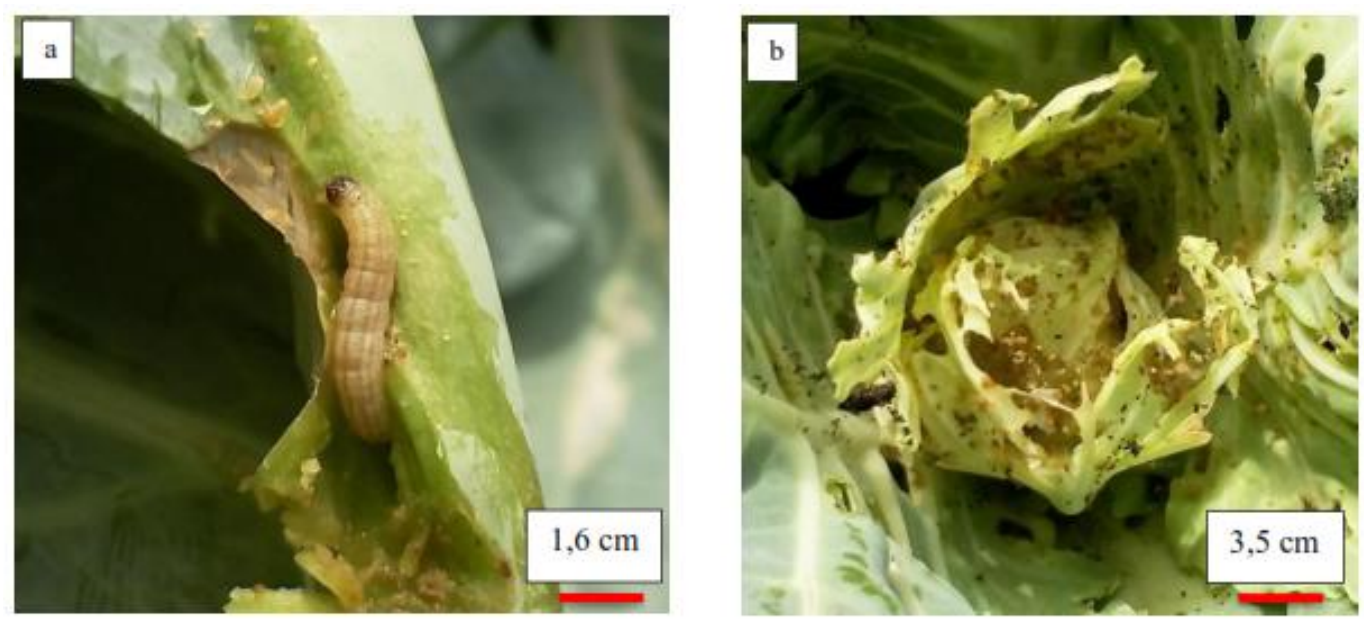

Figure 3 : Plants de chou attaqués par Hellula undalis.

a. Larve sortant d'un orifice de la nervure principale ;

b. Feuilles de pomme rongées.

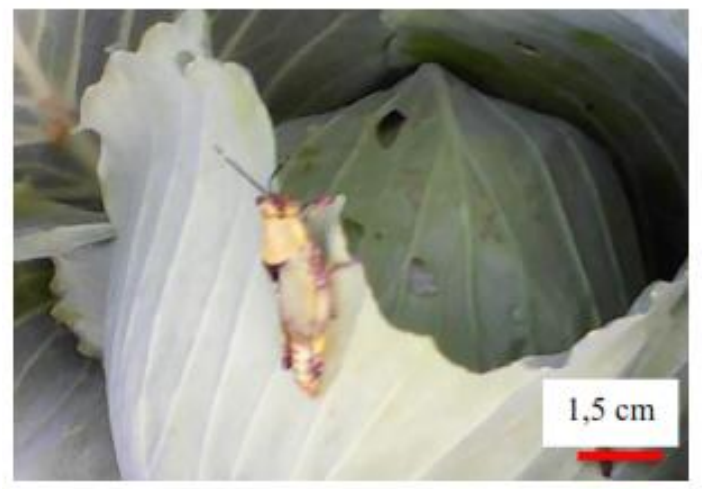

Figure 4: Criquet Zonocerus variegatus consommant une feuille de chou.

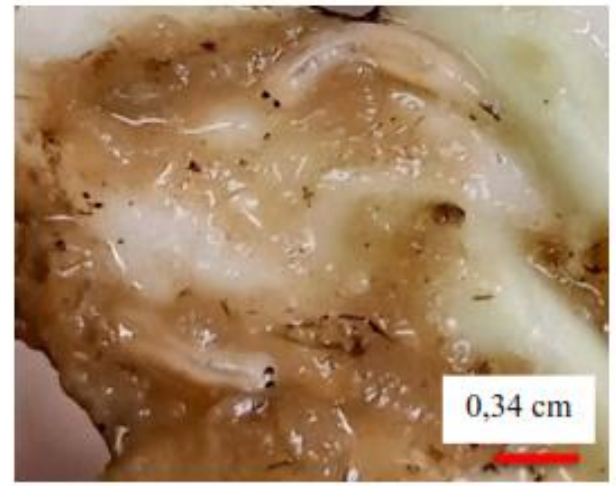

Figure 5: Larves de Diptères sur une feuille de pomme de chou. 
Tableau 1 : Ségrégation des insectes ravageurs du chou en fonction du niveau des attaques.

\begin{tabular}{lrrrrr}
\hline Espèces & \multicolumn{1}{c}{$\mathbf{P 0}$} & \multicolumn{1}{c}{$\mathbf{P 1}$} & $\mathbf{P 2}$ & $\mathbf{P 3}$ & $\mathbf{P 4}$ \\
\hline H. elaterii & $9,18 \pm 8,51 \mathrm{a}$ & $0,81 \pm 0,48 \mathrm{~b}$ & $0 \mathrm{~d}$ & $0 \mathrm{~d}$ & $0 \mathrm{~d}$ \\
P. conica & $8,52 \pm 8,51 \mathrm{a}$ & $1,48 \pm 0,8 \mathrm{~b}$ & $0 \mathrm{~d}$ & $0 \mathrm{~d}$ & $0 \mathrm{~d}$ \\
Z. variegatus & $8,44 \pm 7,44 \mathrm{a}$ & $1,55 \pm 0,6 \mathrm{~b}$ & $0 \mathrm{~d}$ & $0 \mathrm{~d}$ & $0 \mathrm{~d}$ \\
Trichoplusia $n i$ & $9,18 \pm 9,25 \mathrm{a}$ & $0,44 \pm 0,9 \mathrm{c}$ & $0,07 \pm 0,13 \mathrm{~d}$ & $0,15 \pm 0,06 \mathrm{c}$ & $0,15 \pm 0,06 \mathrm{c}$ \\
Sarcophaga sp & $9,37 \pm 3,70 \mathrm{a}$ & $0 \mathrm{~d}$ & $0 \mathrm{~d}$ & $0 \mathrm{~d}$ & $0,63 \pm 0,23 \mathrm{c}$ \\
Lucilia sp & $9,26 \pm 2,22 \mathrm{a}$ & $0 \mathrm{~d}$ & $0 \mathrm{~d}$ & $0 \mathrm{~d}$ & $0,74 \pm 0,35 \mathrm{c}$ \\
H. illucens & $9,22 \pm 2,22 \mathrm{a}$ & $0 \mathrm{~d}$ & $0 \mathrm{~d}$ & $0 \mathrm{~d}$ & $0,78 \pm 0,21 \mathrm{c}$ \\
M. domestica & $9,07 \pm 6,14 \mathrm{a}$ & $0 \mathrm{~d}$ & $0 \mathrm{~d}$ & $0 \mathrm{~d}$ & $0,92 \pm 0,16 \mathrm{c}$ \\
M. persicae & $7,63 \pm 4,81 \mathrm{~b}$ & $0 \mathrm{~d}$ & $0 \mathrm{~d}$ & $2,37 \pm 1,26 \mathrm{a}$ & $0 \mathrm{~d}$ \\
L. erysimi & $7,18 \pm 3,70 \mathrm{~b}$ & $0 \mathrm{~d}$ & $0 \mathrm{~d}$ & $2,81 \pm 2,02 \mathrm{a}$ & $0 \mathrm{~d}$ \\
H. phidilealis & $6,33 \pm 3,41 \mathrm{~b}$ & $0,81 \pm 0,4 \mathrm{~b}$ & $1,29 \pm 1,02 \mathrm{~b}$ & $1,18 \pm 0,92 \mathrm{~b}$ & $0,37 \pm 0,11 \mathrm{c}$ \\
C. binotalis & $6,7 \pm 5,19 \mathrm{~b}$ & $0,48 \pm 0,42 \mathrm{c}$ & $0,96 \pm 0,52 \mathrm{c}$ & $1,37 \pm 0,96 \mathrm{~b}$ & $0,48 \pm 0,16 \mathrm{c}$ \\
S.littoralis & $6,03 \pm 4,13 \mathrm{~b}$ & $0,92 \pm 0,18 \mathrm{~b}$ & $1,85 \pm 1,32 \mathrm{a}$ & $0,63 \pm 0,21 \mathrm{c}$ & $0,55 \pm 0,34 \mathrm{c}$ \\
H. undalis & $4,3 \pm 2,96 \mathrm{c}$ & $0,77 \pm 0,18 \mathrm{~b}$ & $1,29 \pm 1,06 \mathrm{~b}$ & $2,33 \pm 1,37 \mathrm{a}$ & $1,29 \pm 0,12 \mathrm{~b}$ \\
P. xylostella & $1,18 \pm 1,9 \mathrm{~d}$ & $2,11 \pm 1,9 \mathrm{a}$ & $1,92 \pm 1,1 \mathrm{a}$ & $2,70 \pm 2,26 \mathrm{a}$ & $2,15 \pm 0,63 \mathrm{a}$ \\
\hline Test de Tukey au seuil de $5 \% . \mathbf{P 0}:$ nombre de pieds sains $; \mathbf{P 1}:$ nombre de pieds présentant des attaques de niveau faible $; \mathbf{P 2}$ & \\
: nombre de pieds présentant des attaques de niveau modéré $; \mathbf{P 3}:$ nombre de pieds présentant des attaques graves $; \mathbf{P 4}:$ nombre &
\end{tabular}

Tableau 2 : Ségrégation des insectes ravageurs du chou en fonction de l'indice d'intensification des dégâts.

\begin{tabular}{lcrrc}
\hline Espèces & $\begin{array}{c}\text { Nombre } \\
\text { d'observation }\end{array}$ & $\begin{array}{c}\text { Plants } \\
\text { sains }(\%)\end{array}$ & $\begin{array}{c}\text { Plants } \\
\text { atta-qués }\end{array}$ & $\begin{array}{c}\text { Indice d'intensification } \\
\text { des dégâts }(\%)\end{array}$ \\
\hline Henosepilachna & 270 & 91,85 & 8,15 & $2,04 \pm 3,73 \mathrm{e}$ \\
Pyrgomorpha conica & 270 & 85,18 & 14,81 & $3,70 \pm 4,92 \mathrm{e}$ \\
Zonocerus variegatus & 270 & 84,44 & 15,55 & $3,89 \pm 4,66 \mathrm{e}$ \\
Trichoplusia ni & 270 & 91,85 & 8,15 & $4,07 \pm 5,00 \mathrm{e}$ \\
Sarcophaga spp & 270 & 93,7 & 6,3 & $6,30 \pm 7,41 \mathrm{~d}$ \\
Lucilia spp & 270 & 92,6 & 7,4 & $7,40 \pm 10,72 \mathrm{~d}$ \\
Hermetia illucens & 270 & 92,22 & 7,77 & $7,78 \pm 9,02 \mathrm{~d}$ \\
Musca domestica & 270 & 90,74 & 9,26 & $9,26 \pm 12,65 \mathrm{~d}$ \\
Myzus persicae & 270 & 76,3 & 23,7 & $17,78 \pm 5,93 \mathrm{c}$ \\
Lipaphis erysimi & 270 & 71,85 & 28,15 & $21,11 \pm 8,91 \mathrm{c}$ \\
Hellula phidilealis & 270 & 63,33 & 36,66 & $21,11 \pm 15,33 \mathrm{c}$ \\
Crocidolomia binotalis & 270 & 67,03 & 32,96 & $21,11 \pm 16,60 \mathrm{c}$ \\
Spodoptera littoralis & 270 & 60,37 & 39,63 & $21,85 \pm 19,47 \mathrm{c}$ \\
Hellula undalis & 270 & 42,96 & 57,04 & $38,89 \pm 21,45 \mathrm{~b}$ \\
Plutella xylostella & 270 & 11,85 & 88,15 & $56,11 \pm 18,87 \mathrm{a}$ \\
\hline
\end{tabular}

Test de Tukey au seuil de 5\%. 


\section{DISCUSSION}

Tous les organes aériens du chou ont été attaqués par les insectes ravageurs. Les dégâts vont de la simple perforation des feuilles à la destruction de la pomme.

La coccinelle $H$. elaterii et les criquets $P$. conica et $Z$. variegatus ont eu un impact faible sur les cultures du chou. La faiblesse de leurs attaques pourrait s'expliquer par le fait que le chou n'est pas une plante hôte pour ces espèces polyphages. Les attaques de ces ravageurs peuvent donc être considérées comme des attaques isolées. En effet, les sites d'étude étant des périmètres maraîchers, l'abondance et la diversité des cultures peuvent expliquer la présence de ces espèces. Nos résultats sont corroborés par les travaux de Gnago et al. (2010), Chougourou et al. (2012) et Obodji et al. (2016) qui ont observé les espèces $Z$. variegatus et $P$. conica sur le gombo, la tomate et l'aubergine. Quant à l'espèce H. elaterii, Assi et al. (2018) l'ont classée constante sur le concombre alors que Kouassi et al. (2019) ont jugé sa présence accessoire sur le chou.

En ce qui concerne les mouches Sarcophaga sp, Lucilia sp, H. illucens et $M$. domestica, elles ont été des ravageurs secondaires ou opportunistes puisqu'elles n'ont provoqué qu'une décomposition secondaire des tissus végétaux. Leur présence a été conditionnée par l'existence d'une pourriture primaire. Leur nuisibilité provient surtout du caractère répugnant qu'offrent la vue des asticots et la souillure de la pomme par l'infiltration des tissus liquéfiés dans les feuilles de celle-ci. Cette identification de ces espèces saprophages sur le chou et leur classification en tant que nuisibles sont les premières du genre.

Les pucerons $M$. persicae et $L$. erysimi sont des ravageurs qui piquent les feuilles pour en sucer la sève. En cas de fortes attaques, les nombreuses piqûres affaiblissent le plant et entrainent un retard de croissance des jeunes plants. Ce rabougrissement des plants pourrait être la manifestation d'une virose causée par le virus de la mosaïque du navet. Ce virus est transmissible aux cultures de chou par les pucerons, selon le mode persistant et semipersistant. Nos résultats rejoignent ceux de Capinera (2001) qui a expliqué que la transmission de virus à la plante représente le risque majeur de la présence des pucerons pour les cultures.

Les dégâts de $S$. littoralis, $C$. binotalis et $H$. phidilealis ont été jugés préjudiciables pour la culture. Toutefois, le niveau moyen d'intensité des dégâts n'en fait pas de redoutables ravageurs. Le faible niveau d'attaque de $S$. littoralis pourrait s'expliquer par le caractère polyphage de cette espèce. Nos observations sont corroborées par les travaux de Douan (2014) et Obodji et al. (2016) qui ont montré qu'en Côte d'Ivoire, S. littoralis se nourrissaient également sur la tomate et l'aubergine. Sa principale plante hôte est le cotonnier mais l'espèce a également été identifiée sur les céréales comme le niébé (Bello et al., 2018). Quant à $H$. phidilealis, elle n'avait pas encore été mentionnée sur les cultures de chou en Côte d'Ivoire et même en Afrique mais plutôt en Amérique du Sud (Gourmel, 2019). Il se peut qu'elle ait été introduite sur le continent africain depuis plusieurs années mais qu'elle ait été confondue avec $H$. undalis, à cause de la similarité de leur mode de vie.

Contrairement aux autres espèces, les larves de $H$. undalis et de $P$. xylostella ont causé les dégâts les plus importants. Ce sont des espèces grégaires, inféodées à leur plante hôte qu'est le chou. Le nombre élevé de plants attaqués par ces ravageurs $(57,04 \%$ pour $H$. undalis et $88,15 \%$ pour $P$. xylostella) pourrait être lié à une forte population de ces derniers. A ce sujet, Kouassi et al. (2019) ont montré que ces deux espèces représentaient les ravageurs les plus nombreux et les plus constants de la culture du chou. L'importance de leurs dégâts pourrait donc s'expliquer par leur pullulation et leur voracité. Nos résultats sont corroborés par ceux de Sall-Sy (2013) qui avait montré que $P$. xylostella pouvait consommer jusqu'à $60 \%$ du feuillage des plants. Zalucki et al. (2012) ont également estimé les dégâts de cette espèce à 
des pertes de récolte pouvant atteindre $90 \%$. Quant aux larves de $H$. undalis, les résultats concernant cette espèce rejoignent ceux de Sall-Sy (2013) qui a estimé ses dégâts à 33\% de perte de production, manifestés par la formation secondaire de choux à plusieurs petites pommes non commercialisables.

Le taux élevé des organes végétatifs attaqués par $P$. xylostella et la destruction du bourgeon apical par $H$. undalis font de ces deux espèces, les principaux ravageurs du chou pomme. La présence des larves de $P$. xylostella sur au moins $10 \%$ des feuilles et la détection d'au moins une larve de $H$. undalis pourraient permettre d'initier une lutte contre ces ravageurs.

\section{Conclusion}

Sur quinze espèces de ravageurs identifiées, seules $P$. xylostella et $H$. undalis ont été responsables de dégâts dont la qualité et l'abondance entraînent des pertes considérables en culture et à la récolte. Sur la base des résultats obtenus, des traitements phytosanitaires contre $P$. xylostella pourraient être enclenchés lorsque les attaques s'étendent à plus de $10 \%$ des organes végétatifs. Par contre, la lutte contre $H$. undalis devrait être précoce et débuter dès l'apparition de la première larve. Ces résultats pourraient contribuer à la détermination des seuils de nuisibilité pour les ravageurs du chou afin de réduire l'usage abusif des insecticides chimiques de synthèse et de préserver la santé des consommateurs, des producteurs et de l'environnement.

\section{CONFLIT D'INTÉRÊTS}

Les auteurs déclarent ne pas avoir de conflits d'intérêts.

\section{CONTRIBUTIONS DES AUTEURS}

SWMONG et AC ont conçu le projet et élaboré le protocole expérimental. SWMONG a contribué à l'interprétation des résultats et à la finition du manuscrit. MAK a effectué les travaux de terrain, analysé les données et rédigé le premier draft de l'article.

\section{REMERCIEMENTS}

Nous remercions les paysans du village de Waraniéné et du quartier Mongaha dans le département de Korhogo pour la mise à disposition de leurs parcelles. Nous exprimons nos sincères gratitudes à l'Université Peleforo Gon Coulibaly (Côte d'Ivoire), pour l'autorisation d'exploitation du carré potager.

\section{RÉFÉRENCES}

Akessé EN. 2014. Lutte contre les insectes ravageurs de la laitue (Lactuca sativa L., Asteracae) en zone urbaine d'Abidjan: pratiques d'utilisation et quantification des pesticides dans les légumes. Mémoire de Master, Université Félix Houphouët-Boigny, Côte d'Ivoire, $57 \mathrm{p}$.

Akessé EN, Ouali-N'Goran S-WM, Tano Y. 2015. Insectes ravageurs du piment Capsicum chinense Jacq. (Solanaceae) à Port-Bouët (Abidjan-Côte d'Ivoire) : pratiques de lutte par les pesticides chimiques. J. Appl. Biosci., 93: 86678674.

DOI: http://dx.doi.org/10.4314/jab.v93i1.1.

Aléne D, Messi J, Quilici S. 2006. Influence de l'ombrage sur la sensibilité des plantules de Ricinodendron heudelotii (Baill.) aux attaques de Diclidoplebia xuani en milieu naturel au Cameroun. Fruits, 61(4): 273280.

DOI: http://dx.doi.org/10.1051/fruits:2006025.

Assi ANM, Aboua LRN, Ossey CL, Tano DKC. 2018. Entomofauna of cucumber Cucumis sativus (L.), damage assessment caused by insect pests in Dabou in south of Côte d'Ivoire. Int. J. Fauna and Biol. Studies, 5(6): 27-34.

Bellmann B. 2015. 450 Insectes. Les indispensables Delachaux et Niestlé : Paris.

Bello S, Babalakoun AO, Zoudjihékpon J, Coulibaly KA. 2018. Diversité de l'entomofaune du niébé (Vigna unguiculata (L.)Walpers) au Nord-Ouest du Bénin. J. Appl. Biosci., 132: 1342413438. DOI : 10.4314 jab.v132i1.4 
BNETD. 2007. Plan stratégique de développement du département de Korhogo: Diagnostique stratégique. BNETD, Abidjan -Côte d'Ivoire.

Capinera JL. 2001. Green Peach Aphid, Myzus persicae (Sulzer) (Insecta: Hemiptera: Aphididae). EENY-222, Entomology and Nematology Department, University of Florida, UF/IFAS Extension, Gainesville, Florida, $9 \mathrm{p}$.

Chougourou DC, Agbaka A, Adjakpa JB, Koutchika RE, Adjalian EJN. 2012. Inventaire préliminaire de l'entomofaune des champs de tomates (Lycopersicon esculentum Mill) dans la Commune de Djakotomey au Bénin. Int. J. Biol. Chem. Sci., 6(4): 1798-1804. DOI: 10.4314/ijbcs.v6i4.34.

Douan BG. 2014. Bioécologie et moyens de lutte contre Spodoptera littoralis boisduval (Lepidoptera: Noctuidae) sur le chou (Brassica oleracea L. (Brassicales : Brassicaceae)) dans les localités de Bingerville et Azaguié au sud de la Côte d'Ivoire. Thèse, Université Nangui Abrogoua, Abidjan, Côte d'Ivoire, 140 p.

Douan BG, Doumbia M, Kouadio D, Kra VM, Dagnogo M. 2013. Comparaison de la dynamique des populations de Spodoptera littoralis (Boisduval) (Lepidoptera : Noctuidae) à celles de deux lépidoptères du chou dans le district d'Abidjan en Côte d'Ivoire. J. Animal Plant Sci., 17(1): 2412-2424.

Gnago JA, Danho M, Atcham-Agneroh T, Fofana IK, Kohou GA. 2010. Efficacité des extraits de neem (Azadirachta indica) et de papayer (Carica papaya) dans la lutte contre les insectes ravageurs du gombo (Abelmoschus esculentus) et du chou (Brassica oleracea) en Côte d'Ivoire. Int. J. Biol. Chem. Sci., 4(4): 953-965. DOI: 10.4314/ijbcs.v4i4.63035

Gourmel C. 2014. Catalogue Illustré des Principaux Insectes Ravageurs et Auxiliaires des Cultures de Guyane. Coopérative BIO SAVANE : Guyane.
Gourmel C. 2019. Les Parasitoïdes Oophages des Pyrales des Cucurbitaceae Diaphania spp. Présents en Guyane. Coopérative BIO SAVANE : Guyane.

Gueye SP, Labou B, Diatté M, Diarra K. 2020. $\mathrm{La}$ mauvaise pratique phytosanitaire, principale source de contamination du chou au Sénégal. Int. J. Biol. Chem. Sci., 14(2): $\quad 539-554 . \quad$ DOI: https://dx.doi.org/10.4314/ijbcs.v14i2.19

Hammes C, Putoa R. 1986. Catalogue des insectes et acariens d'intérêt agricole en Polynésie Française. Entomologie agricole, notes et documents, $\mathrm{N}^{\circ} 02,264$ $\mathrm{p}$.

Koné K, Tuo Y, Coulibaly T, Koua KH. 2019. Entomofauna and phytosanitary practices in cabbages production (Brassica oleracea L. 1753) in the township of Korhogo of Northern Côte d'Ivoire. IOSR J. Agric. and Veterinary Sci., 12(2): 0913. DOI: $10.9790 / 2380-1202010913$

Kouassi AM, Ouali-N'Goran S-WM, Akessé EN, Ehounou PG, Soro YR, Coulibaly A. 2019. Distribution of insects according to the phenological stages of apple cabbage Brassica oleracea var capitata (Brassicales: Brassicaceae) in Korhogo, northern Côte d'Ivoire. Int. J. Fauna Biol. Studies, 6(5): 43-49.

Leclant F. 1999. Les pucerons des plantes cultivées: clefs d'identification 2. Cultures maraîchères. Institut national de la recherche agronomique : Paris, France, $98 \mathrm{p}$.

Mambe-Ani P, Ouattara KN, Elleingand FE, Kadjo V. 2019. Assessment of the impact of pesticide use in urban and periurban agriculture in Abidjan, Côte d'Ivoire. Int. J. Biol. Chem. Sci., 13(6): 2824-2837. DOI: 10.4314/ijbcs.v13i6.32

Obodji A, Aboua LRN, Tano DKC, Seri Kouassi BP. 2016. Inventory of entomofauna associated with african eggplant (Solanum aethiopicum 1.) According to the phenological stages and assessment of damages caused by insect 
pests. J. Adv. Stud. Agri. Biol. Environ. Sci., 3(2): 12-21.

Ramel A. 2016. Les insectes. Le monde des insectes. www.aramel.free.fr/. 26/07/2016.

Sall-Sy D. 2013. Point sur le suivi des ravageurs du chou dans les Niayes. ISRA/ $\mathrm{CDH} / \mathrm{UCAD} / \mathrm{AUMN}$, Sénégal, $25 \mathrm{p}$.

Soro G, Koffi NM, Brama K, Kouakou YE, M'Bra KR, Soro PD, Soro N. 2018. Utilisation de produits phytosanitaires dans le maraîchage autour du barrage d'alimentation en eau potable de la ville de Korhogo (nord de la Côte d'Ivoire) : risques pour la santé publique. Environ., Risques \& Santé, 17(2): 155-163. DOI : 10.1684/ers.2018.1147

Tano B, Abo K, DembéléA, Fondio L. 2012. Systèmes de production et pratiques à risque en agriculture urbaine: cas du maraîchage dans la ville de Yamoussoukro. Int. J. Biol. Chem. Sci., 5(6): 2317-2329. DOI: 10.4314/ijbcs.v5i6.12

Tuo Y, Dago DN, Yapo LM, Koua KH. 2017. Screening of phytosanitary practices in vegetable growth activities northern of Côte D'Ivoire. Int. J. Recent Scientific Research, 8(6): 17396-17402. DOI: http://dx.doi.org/10.24327/ijrsr.2017. 0806.0346.

Tuo Y, Koné K, Koné M, Koua KE. 2019. Relative abundance of aphids in vegetable crops in the market garden areas of Korhogo, Sinematiali and Ferke, northern Côte d'Ivoire. Int. J. Agri. Environ. Bioresearch, 4(2): 129-138.

Wognin AS, Ouffoue KS, Assemand EF, Tano K, Koffi-Nevry R. 2013. Perception des risques sanitaires dans le maraîchage à Abidjan, Côte d'Ivoire. Int. J. Biol. Chem. Sci., 7(5): 1829-1837. DOI: 10.4314/ijbcs.v7i5.4.

Zahradnik J, Chvala M. 1989. La Grande Encyclopédie des Insectes. Grund: Paris.

Zalucki MP, Shabbir A, Silva R, Adamson D, Liu SS, Furlong MJ. 2012. Estimating the economic cost of one of the world's major insect pests, Plutella xylostella: Just how long is a piece of string? J. Econ. Entomol., 105(4): 1115-1129. DOI: 10.1603/EC12107. 